\title{
Effectiveness of electrothermal bipolar vessel-sealing devices versus other electrothermal and ultrasonic devices for abdominal surgical hemostasis: a systematic review
}

\author{
Petra F. Janssen · Hans A. M. Brölmann • \\ Judith A. F. Huirne
}

Received: 31 December 2011/Accepted: 23 March 2012/Published online: 27 April 2012

(C) The Author(s) 2012. This article is published with open access at Springerlink.com

\begin{abstract}
Background Adequate hemostatic techniques are essential for optimal intra- and postoperative results. A number of different hemostatic techniques and devices have been developed over the past few years, but which device should be preferred during laparoscopic and open abdominal procedures?

Methods We conducted a systematic search for randomized controlled trials (RCTs) that compared the effectiveness and costs of vessel-sealing devices with those of other electrothermal or ultrasonic devices in abdominal surgical procedures.

Results Seven RCTs that included 554 patients met the inclusion criteria. Various procedures that used a vesselsealing device $\left(\right.$ LigaSure $\left.^{\mathrm{TM}}\right)(n=264)$ were compared to ultrasonic devices $(n=139)$ and mono- $(n=20)$ or bipolar devices $(n=130)$. LigaSure was favored in two studies with respect to less blood loss, shorter operating time, and lower costs. However, no differences were observed in the other studies. Considering the relatively low number of complications, all hemostatic devices used may be considered relatively safe. None of the studies reported on quality of life or cost effectiveness.

Conclusions Vessel-sealing devices may be considered safe and their use may reduce costs due to reduced blood loss and shorter operating time in some abdominal surgical procedures compared to mono- or bipolar electrothermal devices. Wider-ranging RCTs of sufficient quality that
\end{abstract}

P. F. Janssen ( $\square)$ · H. A. M. Brölmann · J. A. F. Huirne Department of Obstetrics and Gynecology, VU University Medical Center, De Boelelaan 1117, 1181 HV Amsterdam, The Netherlands

e-mail: pfjanssen@hetnet.nl assess (cost) effectiveness are required to make firm conclusions.

Keywords Systematic review - Hemostasis - LigaSure . Ultrasonic devices · Electrothermal devices .

Randomized controlled trials

Hemorrhage is one of the most frequent complications during surgery. To reduce hemorrhage-related morbidity, adequate hemostatic techniques are essential during surgical procedures. As laparoscopic surgeons are well aware, laparoscopy requires a clean operating field so adequate hemostatic techniques are imperative for optimal intra- and postoperative results. In open procedures, conventional mechanical hemostatic techniques that use sutures or clips are the most frequently used methods to control bleeding. After the introduction of laparoscopy, other hemostatic techniques became more important given the difficulties of applying laparoscopic sutures. In addition, laparoscopic suturing is a time-consuming procedure that carries the risk of knot slippage. Different hemostatic techniques and devices have been developed over time, including laparoscopic suture ligation with different knots and knot applicators, the application of clips, and different electrothermal and ultrasonic coagulation techniques. Clips are easily placed but require accurate vessel dissection and have a considerable risk of dislodgement during tissue manipulation. In laparoscopic surgery, mechanical hemostatic techniques are almost completely replaced by coagulation techniques that use mono- or bipolar coagulation (electrical energy converted to thermal energy) and ultrasonic devices (mechanical energy converted to thermal energy). They offer some advantages, including ease of handling and fast hemostasis that does not require the use of foreign bodies 
that should remain in the surgical site. These advantages are not only useful in laparoscopy but also in open procedures, leading to an increase in the implementation of these techniques in open procedures as well.

However, the use of electrothermal and ultrasonic devices carries the risk of damaging the adjacent structures due to an increase in temperature that may cause thermal injury to the surrounding tissue $[1,2]$. This raises the following question: which coagulation device should preferably be used during laparoscopic and open abdominal procedures in terms of (cost) effectiveness and safety aspects?

\section{Coagulating techniques}

Monopolar high-frequency electrical energy is the oldest method used to coagulate vessels [3]; however, it induces local heating and tissue damage, and due to potential capacitive coupling and spark generation, its use is associated with the risk of electrical injury to the bowel and other organs [4]. Conventional bipolar electrosurgical technology is safer than monopolar energy as the current flows between the two electrodes of the instrument and so reduces collateral damage. Bipolar coagulation enables the surgeon to operate at lower currents thereby decreasing the effect on other organs [3].

Ultrasonic technology controls bleeding through the use of ultrasonic vibration. The vibrating blade induces a rapid rise in temperature resulting in the denaturation of proteins to form a coagulum that seals the vessel [5]. This technique produces minimal amounts of smoke, and the depth of penetration of the energy lateral to the intended focus is also minimal [3].

Vessel-sealing technology is a variant of bipolar electrosurgical technology. It is a combination of a precise amount of bipolar electrocoagulation (high current, low voltage) and pressure on the tissue that leads to the denaturation of the collagen and elastin in the vessel walls and a fusion of these into a hemostatic seal [6]. Most vesselsealing devices use a tissue-based feedback program to regulate the dosage of applied energy [2]. Due to this feedback system, less energy is required, and, based on in vitro studies, it is reported that these devices have reduced local tissue damage in comparison to conventional bipolar devices [7]. Potentially, they therefore represent a decreased risk of injury to adjacent structures. However, are vessel-sealing devices indeed more effective than other electrothermal or ultrasonic devices in terms of reduced coagulation time, fewer complications, and lower costs during laparoscopic and open surgical procedures?

The objective of this current review was to report on available literature in a systematic manner with respect to the (cost) effectiveness of vessel-sealing devices in comparison to electrothermal or ultrasonic devices in abdominal surgical procedures.

\section{Materials and methods}

Search strategy

The MEDLINE, EMBASE, and Cochrane Library electronic databases were searched for articles published from 1998 (when the use of a vessel-sealing instrument was first reported) to October 21, 2011 in order to identify all RCTs that compared vessel-sealing with other electrothermal or ultrasonic coagulation techniques in patients who underwent abdominal surgery. Each of the following search terms were used: "vessel sealing" [tiab] OR vesselsealing[tiab] OR "vessel sealer" [tiab] OR "thermo fusion" [tiab] OR "thermal welding" [tiab] OR ligasure*[tiab] OR enseal*[tiab] OR Gyrus*[tiab] OR biclamp*[tiab] OR plasmakinetic*[tiab]. The complete list of search terms is given in the Appendix. All retrieved abstracts, studies, and citations were reviewed (Fig. 1). Reference lists of the acquired articles were cross-checked for additional relevant studies. The search was not limited by publication status

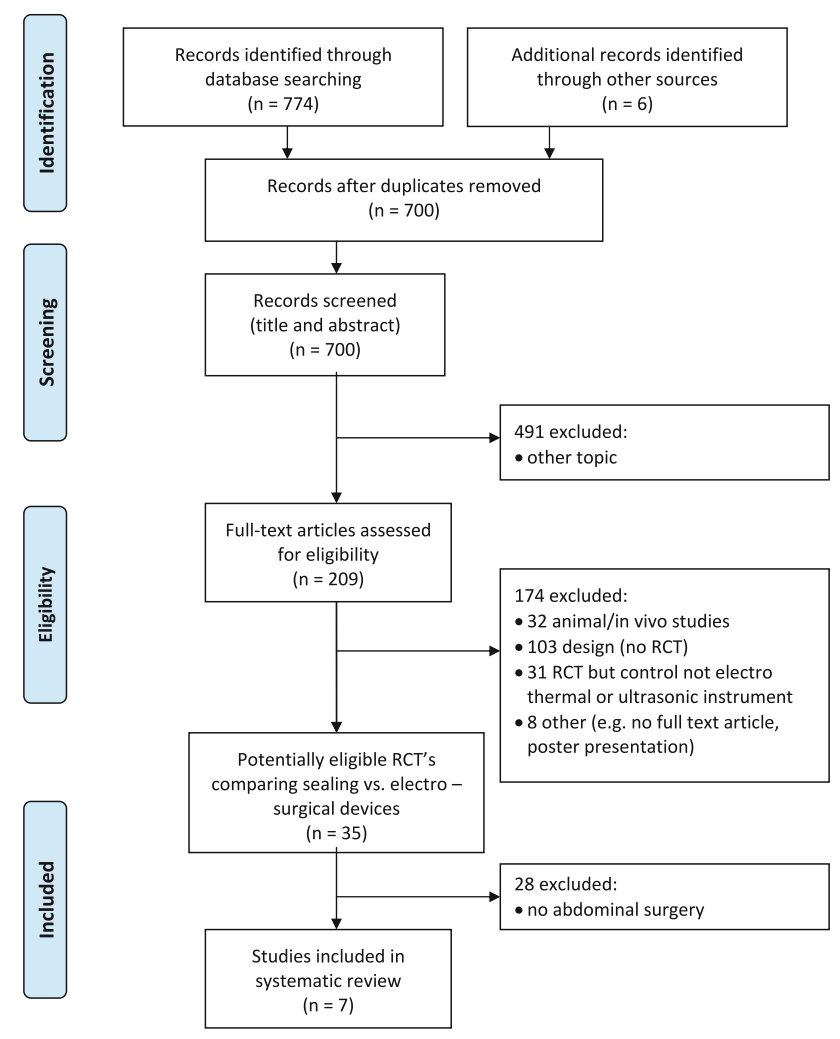

Fig. 1 Flow diagram of reviewed studies (RCT randomized controlled trial) 
and papers had to be written in English or other European languages.

\section{Selection criteria}

To be included in the analysis, studies had to (1) have a randomized controlled design, (2) evaluate a vessel-sealing device versus other electrothermal or ultrasonic hemostatic devices, (3) report on at least one of the outcome measures (e.g., operating time, blood loss, complications, length of hospital stay, quality of life, return to work, costs), and (4) clearly document the operative technique which should have been an abdominal surgical procedure. Studies were excluded from the analysis when it was impossible to extract or calculate the appropriate data from the published results. Furthermore, the methodological quality of the fulltext studies was analyzed following the CONSORT Statement (CONSORT 2010 checklist) [8]. In the event of insufficient or incomplete information, authors were contacted to obtain additional information.

\section{Study selection}

Two reviewers (PJ and $\mathrm{JH}$ ) evaluated each of the eligible studies and decided whether to include or exclude them according to the selection criteria. Differences between the two reviewers were resolved by re-examination of the original article until consensus was attained about the study's data. For trials yielding multiple publications, only the most complete report was included.

\section{Outcome measures}

The following outcomes were used to compare the vesselsealing system with electrothermal or ultrasonic devices in abdominal surgery patients: (1) operative parameters, including operating time and operative blood loss; (2) postoperative parameters comprising length of postoperative hospital stay and return to normal (working) activity; (3) adverse perioperative outcomes and postoperative pain; (4) quality of Life (QOL); and (5) costs.

\section{Data extraction}

Two reviewers (PJ and $\mathrm{JH}$ ) independently extracted the following information from each study: first author, year of publication, study design, study population characteristics, technique of randomization, sample size calculation, surgical and control interventions, number of subjects operated on with each technique, operative parameters (primary or secondary outcomes), adverse outcomes, postoperative recovery, return to work, QOL, and costs.

\section{Results}

Quality and characteristics of eligible studies

The PubMed search yielded 541 titles and EMBASE 233 additional titles. After removing the duplicates, 700 abstracts were studied (Fig. 1). Of these 700 abstracts, 209 papers were identified as being potentially relevant. The hand search of the reviews of the Cochrane Central Register of Controlled Trials and the Register of Controlled Trials did not reveal any additional relevant RCTs or currently running randomized trials. Of the 209 full papers, we identified 35 RCTs that compared vessel-sealing devices with other electrothermal or ultrasonic coagulating devices. Of these 35 RCTs, 28 studies were excluded because they were on nonabdominal surgical procedures such as hemorrhoidectomies.

Seven RCTs [9-15], published between 2005 and 2011, met the selection criteria. The trials contained a total of 554 patients; the largest study was based on 140 patients and the smallest focused on 24 patients. These seven studies reported on various surgical abdominal procedures, including laparoscopic colectomies (three RCTs), hepatic resections, laparoscopic adrenalectomies, laparoscopic hysterectomies, and laparoscopic salpingo-oophorectomies (Table 1).

All trials reported on operating time and blood loss, and most of them reported on short-term recovery parameters and postoperative complications. None of the studies assessed QOL or return to work. Costs were reported in only two studies [9, 10]. None of the studies performed cost-effectiveness or cost-utility analyses.

The methodological quality of the included RCTs was analyzed following the CONSORT criteria; a variety in quality was found among the included RCTs (Table 2). Three trials described in detail the primary and secondary outcome measures, the randomization process, the sample size calculation, and the trial registration number $[9,14$, 15], whereas in two trials the methodological quality was very poorly described [12, 13]. Technique and time of randomization were adequate in six studies. Four studies used computer-generated randomization sequences [10, 11, $14,15]$, one reported on the use of sealed envelopes [9], one "assigned a randomly generated number" [12], and one was described only as "randomized" [13]. Sample size was calculated correctly in five randomized studies, i.e., calculation using the primary outcome measurement (Table 2).

Patients and coagulating devices used

The median sample size of the seven RCTs was 61 patients (range $=24-140)$ (Table 1$)$. In the case of a vessel-sealing 
Table 1 Characteristics of RCTs included in the systematic review

\begin{tabular}{|c|c|c|c|c|c|}
\hline Reference & Procedure & Technique $(n)$ & Additional intervention & Outcome measures & Primary outcome \\
\hline \multirow[t]{4}{*}{$\begin{array}{l}\text { Hubner et al. } \\
2008 \text { [9] }\end{array}$} & \multirow[t]{4}{*}{$\begin{array}{l}\text { Laparoscopic } \\
\text { left-sided } \\
\text { colectomy } \\
(N=61)\end{array}$} & $\begin{array}{l}\text { LigaSure } \\
\quad(n=21) \\
(\text { LigaSure } \\
5 \mathrm{~mm})\end{array}$ & Clips $^{\mathrm{a}}$ & \multirow[t]{4}{*}{$\begin{array}{l}\text { Dissection time, blood loss, } \\
\text { postoperative complications, costs, } \\
\text { practicability of device }\end{array}$} & \multirow[t]{4}{*}{ Dissection time } \\
\hline & & $\begin{array}{l}\text { Ultrasonic } \\
\quad(n=20) \\
(\text { Harmonic Ace })\end{array}$ & Clips $^{\mathrm{a}}$ & & \\
\hline & & $\begin{array}{l}\text { Monopolar } \\
\text { scissors } \\
(n=20)\end{array}$ & Clips $^{\mathrm{a}}$ & & \\
\hline & & $\begin{array}{l}\text { (Endo Shears } \\
5 \mathrm{~mm} \text { ) }\end{array}$ & & & \\
\hline \multirow[t]{4}{*}{$\begin{array}{l}\text { Targarona } \\
\text { et al. } 2005 \\
{[10]}\end{array}$} & \multirow[t]{4}{*}{$\begin{array}{l}\text { Laparoscopic } \\
\text { colectomy } \\
(N=38)\end{array}$} & $\begin{array}{l}\text { LigaSure } \\
\qquad(n=15) \\
(\text { Atlas } 10 \mathrm{~mm})\end{array}$ & None & \multirow[t]{4}{*}{$\begin{array}{l}\text { Operating time, blood loss, } \\
\text { conversion rate, complications, } \\
\text { costs }\end{array}$} & \multirow[t]{4}{*}{ Not mentioned } \\
\hline & & $\begin{array}{l}\text { Ultrasonic } \\
\quad(n=12)\end{array}$ & Endostapler ${ }^{\mathrm{b}}$ & & \\
\hline & & $\begin{array}{l}\text { (Harmonic } \\
\text { Scalpel } 5 \mathrm{~mm} \text { ) }\end{array}$ & & & \\
\hline & & $\begin{array}{l}\text { Conv. } \\
\text { electrosurgery } \\
(n=11)\end{array}$ & Ethicon clip applier ${ }^{\mathrm{b}}$ & & \\
\hline \multirow[t]{3}{*}{$\begin{array}{l}\text { Rimonda } \\
\text { et al. } 2009 \\
\text { [11] }\end{array}$} & \multirow[t]{3}{*}{$\begin{array}{l}\text { Laparoscopic } \\
\text { colectomy } \\
(N=140)\end{array}$} & $\begin{array}{l}\text { LigaSure } \\
(n=70) \\
(\text { LigaSure } \\
10 \mathrm{~mm})\end{array}$ & $\begin{array}{l}\text { In all cases major and } \\
\text { venous vessels were } \\
\text { clipped with } \\
\text { laparoscopic forceps }\end{array}$ & \multirow[t]{3}{*}{$\begin{array}{l}\text { Operative time, blood loss, } \\
\text { morbidity }\end{array}$} & \multirow[t]{3}{*}{$\begin{array}{l}\text { Intraoperative } \\
\text { reduction of blood } \\
\text { loss }\end{array}$} \\
\hline & & $\begin{array}{l}\text { Ultrasonic } \\
\qquad(n=70)\end{array}$ & & & \\
\hline & & $\begin{array}{l}\text { (Ultracision } \\
10 \mathrm{~mm} \text { ) }\end{array}$ & & & \\
\hline \multirow[t]{3}{*}{$\begin{array}{l}\text { Campagnacci } \\
\text { et al. } 2007 \\
{[12]}\end{array}$} & \multirow[t]{3}{*}{$\begin{array}{l}\text { Hepatic } \\
\quad \text { resection } \\
(N=24)\end{array}$} & $\begin{array}{l}\text { LigaSure } \\
\quad(n=12) \\
\text { (LigaSure V) }\end{array}$ & Ligation vessels $>7 \mathrm{~mm}$ & \multirow[t]{3}{*}{$\begin{array}{l}\text { Operating time, blood loss, } \\
\text { morbidity, hospital stay }\end{array}$} & \multirow[t]{3}{*}{ Not mentioned } \\
\hline & & $\begin{array}{l}\text { Ultrasonic } \\
\quad(n=12)\end{array}$ & Ligation vessels $>5 \mathrm{~mm}$ & & \\
\hline & & $\begin{array}{c}\text { (Harmonic } \\
\text { Scalpel) }\end{array}$ & & & \\
\hline \multirow[t]{3}{*}{$\begin{array}{l}\text { Guerrieri } \\
\text { et al. } 2007 \\
{[13]}\end{array}$} & \multirow[t]{3}{*}{$\begin{array}{l}\text { Laparoscopic } \\
\text { adrenalectomy } \\
(N=50)\end{array}$} & $\begin{array}{l}\text { LigaSure } \\
\quad(n=25) \\
\text { (LigaSure V) }\end{array}$ & Titanium clips $^{\mathrm{d}}$ & \multirow[t]{3}{*}{$\begin{array}{l}\text { Operative time, blood loss, } \\
\text { complications, conversion rate, } \\
\text { hospital stay }\end{array}$} & \multirow[t]{3}{*}{ Not mentioned } \\
\hline & & $\begin{array}{l}\text { Ultrasonic } \\
\quad(n=25)\end{array}$ & Titanium clips $^{\mathrm{d}}$ & & \\
\hline & & $\begin{array}{l}\text { (UltraSonic } \\
\text { Shears) }\end{array}$ & & & \\
\hline \multirow[t]{3}{*}{$\begin{array}{l}\text { Janssen et al. } \\
2011[14]\end{array}$} & \multirow[t]{3}{*}{$\begin{array}{l}\text { Laparoscopic } \\
\text { hysterectomy } \\
(N=140)\end{array}$} & $\begin{array}{l}\text { LigaSure } \\
\quad(n=70) \\
(\text { LigaSure Lap } \\
5 \mathrm{~mm})\end{array}$ & None & \multirow[t]{3}{*}{$\begin{array}{l}\text { Operating time, time to dissect } \\
\text { adnexal ligaments, blood loss, } \\
\text { complications, subjective } \\
\text { evaluation devices }\end{array}$} & \multirow[t]{3}{*}{$\begin{array}{l}\text { Operating time until } \\
\text { complete } \\
\text { detachment of the } \\
\text { uterus }\end{array}$} \\
\hline & & $\begin{array}{l}\text { Conv. bipolar } \\
(n=70)\end{array}$ & None & & \\
\hline & & $\begin{array}{l}\text { (Seitzinger or } \\
\text { Cutting } \\
\text { Forceps } \\
5 \mathrm{~mm} \text { ) }\end{array}$ & & & \\
\hline
\end{tabular}


Table 1 continued

\begin{tabular}{|c|c|c|c|c|c|}
\hline Reference & Procedure & Technique $(n)$ & Additional intervention & Outcome measures & Primary outcome \\
\hline \multirow[t]{3}{*}{$\begin{array}{c}\text { Janssen et al. } \\
2012 \text { [15] }\end{array}$} & \multirow[t]{3}{*}{$\begin{array}{l}\text { Laparoscopic } \\
\text { salpingo- } \\
\text { oophorectomy } \\
(N=100)\end{array}$} & $\begin{array}{l}\text { LigaSure } \\
\quad(n=51) \\
(\text { LigaSure Lap } \\
\quad 5 \mathrm{~mm})\end{array}$ & None & \multirow[t]{3}{*}{$\begin{array}{l}\text { Operating time, blood loss, } \\
\text { complications, subjective } \\
\text { evaluation devices }\end{array}$} & \multirow[t]{3}{*}{$\begin{array}{l}\text { Operating time until } \\
\text { complete } \\
\text { detachment of the } \\
\text { specimen }\end{array}$} \\
\hline & & $\begin{array}{l}\text { Conv. bipolar } \\
(n=49)\end{array}$ & None & & \\
\hline & & $\begin{array}{l}\text { (Seitzinger or } \\
\quad \text { Cutting } \\
\text { Forceps } \\
5 \mathrm{~mm} \text { ) }\end{array}$ & & & \\
\hline
\end{tabular}

${ }^{a}$ Clips were used for ligation of large vessels (not further specified)

b In the ultrasonic group, the mesenteric vessel pedicle was controlled with an endostapler; in the conventional electrosurgery group, the largest vessels, including the inferior mesenteric artery, were controlled with clips

c Monopolar and bipolar coagulation were used in both groups when preferred and deemed necessary

${ }^{\mathrm{d}}$ When the adrenal vein was estimated to measure over $5 \mathrm{~mm}$, clips were used, but this use was not further specified in the ultrasonic group

Table 2 Characteristics of RCTs according to some of the CONSORT Statement criteria

\begin{tabular}{|c|c|c|c|c|}
\hline RCT [ref.] & Randomization process & Sample size calculation & $\begin{array}{l}\text { Trial } \\
\text { registration } \\
\text { no. }\end{array}$ & Conflict of interest \\
\hline $\begin{array}{l}\text { Laparoscopic left- } \\
\text { sided colectomy } \\
(N=61)[9]\end{array}$ & Sealed envelopes & $\begin{array}{l}20 \text { Patients in each group to find a } 30 \text {-min } \\
\text { reduction in dissection time }\end{array}$ & Registered & $\begin{array}{l}\text { No financial } \\
\text { support received } \\
\text { from } \\
\text { manufacturers }\end{array}$ \\
\hline $\begin{array}{l}\text { Laparoscopic } \\
\text { colectomy } \\
(N=38)[10]\end{array}$ & $\begin{array}{l}\text { Computer-generated random } \\
\text { numbers }\end{array}$ & $\begin{array}{l}12 \text { Per group required for a reduction of } \\
30 \% \text { of operative time }\end{array}$ & $\begin{array}{l}\text { No } \\
\text { registration }\end{array}$ & Not mentioned \\
\hline $\begin{array}{l}\text { Laparoscopic } \\
\text { colectomy } \\
(N=140)[11]\end{array}$ & $\begin{array}{l}\text { Sealed opaque envelopes containing } \\
\text { computer-generated random } \\
\text { numbers }\end{array}$ & $\begin{array}{l}140 \text { Patients sufficient to prove a difference } \\
\text { of } 20 \% \text { in blood loss }\end{array}$ & $\begin{array}{l}\text { No } \\
\text { registration }\end{array}$ & Not mentioned \\
\hline $\begin{array}{l}\text { Hepatic resection } \\
\quad(N=24)[12]\end{array}$ & Randomly generated number & Not before starting the trial & $\begin{array}{l}\text { No } \\
\text { registration }\end{array}$ & Not mentioned \\
\hline $\begin{array}{l}\text { Laparoscopic } \\
\text { adrenalectomy } \\
(N=50)[13]\end{array}$ & Not specified & Not mentioned & $\begin{array}{l}\text { No } \\
\text { registration }\end{array}$ & Not mentioned \\
\hline $\begin{array}{l}\text { Laparoscopic } \\
\text { hysterectomy } \\
(N=140)[14]\end{array}$ & $\begin{array}{l}\text { Web-based central computer system } \\
\text { generated study numbers and } \\
\text { randomization outcome }\end{array}$ & $\begin{array}{l}130 \text { Patients required to detect } 20 \% \\
\text { reduction in operating time till complete } \\
\text { detachment of the uterus }\end{array}$ & Registered & $\begin{array}{l}\text { No financial } \\
\text { support }\end{array}$ \\
\hline $\begin{array}{l}\text { Laparoscopic } \\
\text { salpingo- } \\
\text { oophorectomy } \\
(N=100)[15]\end{array}$ & $\begin{array}{l}\text { Web-based central computer system } \\
\text { generated study numbers and } \\
\text { randomization outcome }\end{array}$ & $\begin{array}{l}97 \text { Patients required to detect a } 15-\mathrm{min} \\
\text { reduction in operating time till complete } \\
\text { detachment of the specimen }\end{array}$ & Registered & $\begin{array}{l}\text { No financial } \\
\text { support }\end{array}$ \\
\hline
\end{tabular}

device, LigaSure ${ }^{\mathrm{TM}}$ (Valleylab, Boulder, CO, USA) was the only device used. None of the published RCTs included any other type of vessel-sealing device. In $48.1 \%$ of the cases, a LigaSure device $(n=264)$ was compared with ultrasonic devices $(n=139)$, in $45.0 \%$ with conventional bipolar devices $(n=130)$, and in $6.9 \%$ with monopolar devices $(n=20)$. LigaSure $10-\mathrm{mm}$ devices were used in the study of Targarona et al. [10] and Rimonda et al. [11] and 5-mm devices were used in the other five studies (Table 1). The surgical procedure technique was described in detail in all seven RCTs. Five of the seven RCTs allowed additional interventions to control hemostasis. The criteria for using these additional interventions were described precisely only in the studies of Targarona et al. [10] and Campagnacci et al. [12]. In the remaining three studies, the criteria were not sufficiently described. 
Table 3 Clinical results extracted from the RCTs included in the systematic review

\begin{tabular}{|c|c|c|c|c|c|c|}
\hline Procedure [ref.] & Technique & $\begin{array}{l}\text { Operating time } \\
(\min )\end{array}$ & $\begin{array}{l}\text { No. of sutures } \\
\text { or clips }\end{array}$ & $\begin{array}{l}\text { Blood loss } \\
(\mathrm{mL})\end{array}$ & $\begin{array}{l}\text { Hospital stay } \\
\text { (days) }\end{array}$ & $\operatorname{Costs}^{\mathrm{a}}(€)$ \\
\hline \multirow[t]{4}{*}{ Laparoscopic colectomy $(N=61)$ [9] } & LigaSure $(n=21)$ & 105 & $0(0-3)$ & 50 & 7 & 1,181 \\
\hline & Ultrasonic $(n=20)$ & 90 & $3(0-11)$ & 50 & 7 & 1,115 \\
\hline & $\begin{array}{l}\text { Monopolar scissor } \\
\quad(n=20)\end{array}$ & 137 & $9(4-28)$ & 125 & 8.5 & 1,327 \\
\hline & & $p<0.001^{\mathrm{b}}$ & $p<0.001^{\mathrm{c}}$ & $p=0.22$ & $p=0.45$ & $p=0.009^{\mathrm{d}}$ \\
\hline \multirow[t]{4}{*}{ Laparoscopic colectomy $(N=38)[10]$} & LigaSure $(n=15)$ & 110 & NA & 100 & 6 & 2,664 \\
\hline & Ultrasonic $(n=12)$ & 120 & NA & 100 & 8 & 2,938 \\
\hline & $\begin{array}{l}\text { Electrosurgery } \\
\quad(n=11)\end{array}$ & 180 & NA & 200 & 7 & 2,995 \\
\hline & & $p=0.01^{\mathrm{b}}$ & & $p<0.01^{*}$ & NS & NS \\
\hline \multirow{2}{*}{$\begin{array}{l}\text { Laparoscopic colectomy }(N=140) \\
\text { [11] }\end{array}$} & LigaSure $(n=70)$ & 116.3 & NA & 111.2 & 6.9 & \\
\hline & Ultrasonic $(n=70)$ & $\begin{array}{l}114.8 \\
p=0.89\end{array}$ & NA & $\begin{array}{l}107.9 \\
p=0.72\end{array}$ & $\begin{array}{l}7.4 \\
p=0.37\end{array}$ & NA \\
\hline \multirow[t]{2}{*}{ Hepatic resection $(N=24)[12]$} & LigaSure $(n=12)$ & 136.9 & NA & 210 & 6.1 & \\
\hline & Ultrasonic $(n=12)$ & $\begin{array}{l}183.6 \\
p=0.08\end{array}$ & NA & $\begin{array}{l}485 \\
p<0.05\end{array}$ & $\begin{array}{l}7.8 \\
p>0.05\end{array}$ & NA \\
\hline \multirow{3}{*}{$\begin{array}{l}\text { Laparoscopic adrenalectomy }(N=50) \\
\text { [13] }\end{array}$} & LigaSure $(n=25)$ & left/right & 4 & 83 & 2.9 & \\
\hline & Ultrasonic $(n=25)$ & $\begin{array}{l}51.8 / 72.2 \\
68.6 / 94\end{array}$ & 21 & 210 & 3.1 & NA \\
\hline & & $\mathrm{NS} / p=0.02$ & & $p<0.05$ & NS & \\
\hline \multirow{3}{*}{$\begin{array}{l}\text { Laparoscopic hysterectomy }(N=140) \\
\text { [14] }\end{array}$} & LigaSure $(n=70)$ & 97.6 & 0 & 234.1 & 2.9 & \\
\hline & $\begin{array}{l}\text { Conv. bipolar } \\
(n=70)\end{array}$ & 91.8 & 0 & 273.1 & 2.9 & NA \\
\hline & & $p=0.39$ & & $p=0.46$ & $p=0.94$ & \\
\hline \multirow{3}{*}{$\begin{array}{l}\text { Laparoscopic salpingo-oophorectomy } \\
\qquad(N=100)[15]\end{array}$} & LigaSure $(n=51)$ & 41.0 & 0 & 38.0 & 1.3 & \\
\hline & $\begin{array}{l}\text { Conv. bipolar } \\
(n=49)\end{array}$ & 39.2 & 0 & 33.3 & 1.2 & NA \\
\hline & & $p=0.78$ & & $p=0.73$ & $p=0.89$ & \\
\hline
\end{tabular}

All outcome results are means, except for the study of Hubner et al. [9] and Targarona et al. [10] the results of which are medians $N S$ not significant, $N A$ not analyzed

$* p<0.01$ Ultracision versus bipolar electrosurgery

${ }^{\text {a }}$ Costs per procedure, including the operating room costs, plus the disposable devices costs

b Median dissection time significantly shorter with LigaSure or ultrasonic device than with mono- or bipolar electrosurgery

c More clips were required in the monopolar electrosurgery scissor group than in the other two groups

d Colonic dissection by LigaSure and Ultracision was less expensive than by monopolar electroscissor when a center's volume of 200 patients per year was assumed

\section{Operative results}

The clinical results of the seven RCTs are presented in Table 3.

\section{Operating time}

In two of the laparoscopic colectomy RCTs, operating time was significantly shorter when the LigaSure device was used compared to monopolar electroscissor and bipolar electrothermal devices $[9,10]$. In the laparoscopic adrenalectomy study, the mean operating time was approximately $20 \mathrm{~min}$ shorter per side with LigaSure versus Ultracision, with the difference statistically significant on the left side [13]. In the hepatic resection study there was a trend toward shorter mean operating time (46.7 min shorter, $p=0.08$ ) with LigaSure versus Ultracision [12]. No differences in operating time were reported when comparing the LigaSure device with conventional bipolar devices in both laparoscopic hysterectomies and laparoscopic salpingo-oophorectomies $[14,15]$. However, in one of these studies [14] the reported transection time of the adnexal pedicles was significantly shorter. In the studies of Hubner et al. [9] and Guerrieri et al [13], more clips and sutures were used in the control groups. 
Table 4 Perioperative complications extracted from the included RCTs

\begin{tabular}{|c|c|c|c|c|}
\hline Procedure [ref.] & Technique & $\begin{array}{l}\text { Minor }^{\mathrm{a}} \\
\text { complications }\end{array}$ & $\begin{array}{l}\text { Major }^{\mathrm{b}} \\
\text { complications }\end{array}$ & $\begin{array}{l}\text { Total } \\
\text { complications }\end{array}$ \\
\hline \multirow[t]{4}{*}{ Laparoscopic colectomy $(N=61)$ [9] } & LigaSure $(n=21)$ & 5 & 5 & $10 / 21$ \\
\hline & Ultrasonic $(n=20)$ & 6 & 0 & $6 / 20$ \\
\hline & $\begin{array}{l}\text { Monopolar scissors } \\
\quad(n=20)\end{array}$ & 7 & 3 & $10 / 20$ \\
\hline & & & & $p=0.38$ \\
\hline \multirow[t]{4}{*}{ Laparoscopic colectomy $(N=38)[10]$} & LigaSure $(n=15)$ & & & $2 / 15$ \\
\hline & Ultrasonic $(n=12)$ & NR & NR & $2 / 11$ \\
\hline & $\begin{array}{l}\text { Conv. electrosurgery } \\
\quad(n=11)\end{array}$ & & & $4 / 11$ \\
\hline & & & & NS \\
\hline \multirow[t]{2}{*}{ Laparoscopic colectomy $(N=140)[11]$} & LigaSure $(n=70)$ & 2 & 8 & $10 / 70$ \\
\hline & Ultrasonic $(n=70)$ & 1 & 8 & $9 / 70$ \\
\hline \multirow[t]{2}{*}{ Hepatic resection $(N=24)[12]$} & LigaSure $(n=12)$ & 0 & - & 0 \\
\hline & Ultrasonic $(n=12)$ & 3 & - & 3 \\
\hline \multirow[t]{2}{*}{ Laparoscopic adrenalectomy $(N=50)[13]$} & LigaSure $(n=25)$ & 1 & - & $1 / 25$ \\
\hline & Ultrasonic $(n=25)$ & 1 & - & $1 / 25$ \\
\hline \multirow[t]{3}{*}{ Laparoscopic hysterectomy $(N=140)[14]$} & LigaSure $(n=70)$ & 3 & 4 & $7 / 70$ \\
\hline & Conv. bipolar $(n=70)$ & 6 & 4 & $10 / 70$ \\
\hline & & & & NS \\
\hline \multirow{3}{*}{$\begin{array}{l}\text { Laparoscopic salpingo-oophorectomy }(N=100) \\
\text { [15] }\end{array}$} & LigaSure $(n=51)$ & 3 & - & $3 / 51$ \\
\hline & Conv. bipolar $(n=49)$ & 2 & - & $2 / 49$ \\
\hline & & & & NS \\
\hline
\end{tabular}

$N R$ not registered

${ }^{a}$ No need for specific intervention or requiring specific pharmacological treatment

b Requiring surgical, endoscopic, or radiological intervention; or life-threatening complications requiring intensive care; or death from complication

\section{Blood loss}

Total blood loss was less with the use of LigaSure devices in comparison with ultrasonic devices during laparoscopic adrenalectomies and hepatic resections [12, 13], whereas the other RCTs found no differences in registered blood loss (Table 3).

Adverse outcomes and hospital stay

All seven RCTs documented their perioperative complications and adverse outcomes. The peri- and postoperative complications, which were well described in the RCTs, ranged from minor (e.g., urine retention, wound infection) to major complications (e.g., bowel perforation, postoperative death as a consequence of an anastomotic leakage and subsequent sepsis). We used the subdivision of complications into minor and major as previously defined in a study protocol of Bijen et al. [16]. A complication occurred in 33 $(12.5 \%)$ of the total 264 patients in whom a LigaSure device was used. In total, 47 (16.3\%) complications occurred in the other 289 control patients in whom conventional mono- or bipolar electrothermal or ultrasonic devices were used (Table 4).

The conversion rate to open surgery was described in five studies; there were nine cases in the LigaSure group, six in the ultrasonic group, three in the conventional bipolar device group, and one in the monopolar device group. No significant differences were reported among the different devices used.

The mean difference in length of hospital stay among the various studies did not exceed 1 day, nor did it reach statistical significance in any of the procedures. None of the studies assessed quality of life or return to work (RTW).

The perioperative costs were calculated in two studies, both studying laparoscopic colectomy [9, 10]. Mean calculated costs per procedure, including operating room costs and the costs of disposable devices, were lower using LigaSure or ultrasonic devices compared with monopolar scissors in one study [9] (Table 3). However, these lower costs were not confirmed in a smaller study that compared LigaSure with ultrasonic or bipolar electrosurgical devices [10]. Cost-effectiveness or utility analyses were not performed. 


\section{Discussion}

Our systematic review focused on the effectiveness of vessel-sealing devices compared to other electrothermal or ultrasonic coagulating devices during abdominal surgical procedures. Our search of the literature resulted in seven randomized controlled trials that met our criteria.

Currently, two systematic reviews have already been published comparing LigaSure devices with other hemostatic devices. One studied the effects of various coagulating devices during hemorrhoidectomy and found in favor the LigaSure devices in terms of patient tolerance [17]. The other published systematic review compared LigaSure devices with any coagulating technique, including mechanical techniques, in laparoscopic colectomies [18]. Both systematic reviews provided valid evidencebased information on the potential advantages of LigaSure in comparison to conventional techniques and mechanical devices, but they did not provide sufficient information to make evidence-based conclusions and to base the choice of a specific electrothermal or ultrasonic device to be used during laparoscopic or open abdominal surgery in terms of (cost) effectiveness. This is why in our systematic review we included only studies that compared a vessel-sealing device with another electrothermal or ultrasonic device during abdominal surgery. Despite the fact that we included all types of abdominal surgery and all types of vesselsealing devices, only seven RCTs met all of the selection criteria. The only vessel-sealing devices used in the RCTs were LigaSure devices. So far no published RCTs on abdominal surgery in which Enseal, Bicision, or Gyrus devices were used. Given the extensive number of abdominal surgical procedures performed worldwide in which one type of vessel-sealing device was used, this number of RCTs that met our criteria is surprisingly low. One reason commonly given for the lack of RCT data for these devices is that clinicians require specific training to be able to properly use these devices, which exposes the risk of including a learning curve in one of the arms and consequently the introduction of a potential confounding factor. Another challenge is the rapid evolution of devices, which makes it difficult to determine the optimal time for a clinical study because by the time the study is completed, the device's features have been improved [19]. However, in our opinion new devices should be compared in a random way to other hemostatic devices before they are used in daily practice. Due to the low number of RCTs included, it was not possible to generate homogeneous patient groups with comparable characteristics within a group allowing meta-analyses of the results. Based on the heterogeneity of surgical procedures performed and the control groups, the interpretation of the results is a delicate task and generalization of the conclusions for all surgical procedures and populations should be prevented. Some advantages were reported in the vessel-sealing arms compared to other coagulating devices in terms of reduced operating time and blood loss [9, 10, 12, 13]. The observed difference in operating time might be explained by more efficient coagulation, which is in line with the faster transection time of ovarian vessels observed in one study [14] and the lower number of additional clip applications in some other studies $[9,12,13]$. Furthermore, due to reduced blood loss, the need for fluid suction and rinsing will be reduced which saves time. However, shorter operating times were not reported in all studies $[14,15]$. Some explanations for these discrepancies in outcome can be postulated. First, the power of some studies might not have been sufficient to observe significant differences. Second, the learning curve for the different devices used may have been different between the two arms and was not separately recorded in the studies. Third, in some RCTs additional clip or vessel ligation was allowed in the event of insufficient coagulation results [9-13]. The application of this cointervention might be a confounder which was less often applied in the LigaSure arms. Some of these studies gave clear criteria for the application of these cointerventions [10, 12], but none corrected for this cointervention during the statistical analyses. Finally, a clear definition of operating time was not always described and in some cases might have included nonsurgical time as well. Reporting bias for surgery time or blood loss due to the expectations of the surgeons cannot be excluded. In addition, most studies did not report the exact method of total blood loss calculation. During surgery, particularly during laparoscopic procedures, rinsing is often applied, making the exact estimation of total blood loss imprecise, except if the total rinsed fluid and collected fluid in the suction containers are exactly calculated. Thus, the lack of exact blood loss calculations introduces an additional risk of bias. Furthermore, only three trials reported their disclosure of interest $[9,14,15]$ so we cannot exclude influences of concerned manufacturers on the results.

Only two of the included studies reported on cost data [9, 10]. A significant calculated cost reduction was reported in one study in favor of the LigaSure or ultrasonic device in comparison to the monopolar scissor [9]. In the other study, the cost reduction marked a trend favoring the LigaSure device; however, it was not statistically significant [10]. As a result of mainly shorter operating times, the use of these devices resulted in lower total hospital costs despite the higher costs of the disposable devices used. As related costs and use of materials differ considerably between countries and even hospitals, the cost analyses should be interpreted with caution and these calculations will probably be beneficial only if large numbers of surgical procedures are performed per year. Despite the growing interest in the cost 
effectiveness of endoscopic surgery, none of the RCTs performed an analysis of cost effectiveness.

The relatively low number of reported complications and, in particular, major complications indicates that all coagulating devices are relatively safe. However, the sample size calculations of the included RCTs were not sufficient enough to compare complication rates in relation to the devices used. In addition, due to the small number of the patients included in this review, it is not possible to identify any potential reducing effect on the thermal spread-related complications of vessel-sealing devices compared to other electrothermal or ultrasonic devices.

Finally, we minimized potential bias by performing a precise search for published studies through the use of explicit methods for study selection and data extraction. However, the quality of the studies was not always as good as a RCT should be in terms of the CONSORT Statement criteria. Despite the extensive number of papers reporting on LigaSure devices, most clinical trials are not randomized and often not controlled. Therefore, we have to conclude that yet more well-designed studies are needed before giving appropriate advice on the preferred hemostatic device for abdominal surgery in terms of hemostatic effect, complications, and cost effectiveness. These studies should be sufficiently well designed and preferably should assess, besides surgical outcome parameters, QOL, recovery, return to work, and direct and indirect costs. Vesselsealing devices seem to be as safe as other electrothermal or ultrasonic devices and their use may reduce costs due to reduced blood loss and operating time in some abdominal surgical procedures in comparison to monopolar electrothermal devices. However, these results should be confirmed in larger studies before making firm conclusions.

Acknowledgments We thank $\mathrm{H}$. Ket for his help and advice how to perform a systematic literature search.

Disclosures P. F. Janssen, H. A. M. Brölmann, and J. A. F. Huirne have no conflicts of interest or financial ties to disclose.

Open Access This article is distributed under the terms of the Creative Commons Attribution License which permits any use, distribution, and reproduction in any medium, provided the original author(s) and the source are credited.

\section{Appendix: complete list of search terms}

"vessel sealing" [tiab] OR vesselsealing[tiab] OR "vessel seal" [tiab] OR ligasure[tiab] OR enseal*[tiab] OR ebvs[tiab] OR "vessel sealer" [tiab] OR ligasure*[tiab] OR Gyrus*[tiab] OR thermofusion[tiab] OR "thermo fusion"[tiab] OR Erbe[tiab] OR erbe*[tiab] OR "thermal welding"[tiab] OR plasmakinetic*[tiab] OR marseal*[tiab]
OR GyrusPK[tiab] OR "Gyrus PK*"[tiab] OR sealsafe*[tiab] OR "seal safe" [tiab] OR endoseal*[tiab] OR biclamp*[tiab] OR "bi clamp"[tiab] OR "tissue seal”[tiab] OR thermostapler*[tiab] OR "thermo stapler*"[tiab].

\section{References}

1. Kim FJ, Chammas MFJ, Gewehr E, Morihisa M, Caldas F, Hayacibara E et al (2008) Temperature safety profile of laparoscopic devices: Harmonic ACE (ACE), Ligasure V (LV), and plasma trisector (PT). Surg Endosc 22(6):1464-1469

2. Heniford BT, Matthews BD, Sing RF, Backus C, Pratt B, Greene FL (2001) Initial results with an electrothermal bipolar vessel sealer. Surg Endosc 15(8):799-801

3. Lantis JC II, Durville FM, Connolly R, Schwaitzberg SD (1998) Comparison of coagulation modalities in surgery. J Laparoendosc Adv Surg Tech A 8(6):381-394

4. Harrell AG, Kercher KW, Heniford BT (2004) Energy sources in laparoscopy. Semin Laparosc Surg 11(3):201-209

5. Noble EJ, Smart NJ, Challand C, Sleigh K, Oriolowo A, Hosie KB (2011) Experimental comparison of mesenteric vessel sealing and thermal damage between one bipolar and two ultrasonic shears devices. Br J Surg 98(6):797-800

6. Kennedy JS, Stranahan PL, Taylor KD, Chandler JG (1998) High-burst-strength, feedback-controlled bipolar vessel sealing. Surg Endosc 12(6):876-878

7. Landman J, Kerbl K, Rehman J, Andreoni C, Humphrey PA, Collyer W et al (2003) Evaluation of a vessel sealing system, bipolar electrosurgery, harmonic scalpel, titanium clips, endoscopic gastrointestinal anastomosis vascular staples and sutures for arterial and venous ligation in a porcine model. J Urol 169(2):697-700

8. Schulz KF, Altman DG, Moher D (2010) CONSORT 2010 statement: updated guidelines for reporting parallel group randomised trials. BMJ 340:c332

9. Hubner M, Demartines N, Muller S, Dindo D, Clavien PA, Hahnloser D (2008) Prospective randomized study of monopolar scissors, bipolar vessel sealer and ultrasonic shears in laparoscopic colorectal surgery. Br J Surg 95(9):1098-1104

10. Targarona EM, Balague C, Marin J, Neto RB, Martinez C, Garriga J et al (2005) Energy sources for laparoscopic colectomy: a prospective randomized comparison of conventional electrosurgery, bipolar computer-controlled electrosurgery and ultrasonic dissection. Operative outcome and costs analysis. Surg Innov 12(4):339-344

11. Rimonda R, Arezzo A, Garrone C, Allaix ME, Giraudo G, Morino M (2009) Electrothermal bipolar vessel sealing system vs. harmonic scalpel in colorectal laparoscopic surgery: a prospective, randomized study. Dis Colon Rectum 52(4):657-661

12. Campagnacci R, De Sanctis A, Baldarelli M, Di Emiddio M, Organetti L, Nisi M et al (2007) Hepatic resections by means of electrothermal bipolar vessel device (EBVS) LigaSure V: early experience. Surg Endosc 21(12):2280-2284

13. Guerrieri M, Crosta F, De Sanctis A, Baldarelli M, Lezoche G, Campagnacci R (2008) Use of the electrothermal bipolar vessel system (EBVS) in laparoscopic adrenalectomy: a prospective study. Surg Endosc 22(1):141-145

14. Janssen P, Brölmann H, van Kesteren P, Bongers M, Thurkow A, Heymans $M$ et al (2011) Perioperative outcomes using LigaSure compared with conventional bipolar instruments in laparoscopic hysterectomy: a randomised controlled trial. BJOG 118(13): $1568-1575$ 
15. Janssen $\mathrm{P}$, Brölmann $\mathrm{H}$, van Kesteren $\mathrm{P}$, Bongers $\mathrm{M}$, Thurkow A, Heymans M et al (2012) Perioperative outcomes using LigaSure compared with conventional bipolar instruments in laparoscopic salpingo-oophorectomy: a randomized controlled trial. Surg Endosc. doi:10.1007/s00464012-2274-8

16. Bijen CBM, Briet JM, de Bock GH, Arts HJG, Bergsma-Kadijk JA, Mourits MJE (2009) Total laparoscopic hysterectomy versus abdominal hysterectomy in the treatment of patients with early stage endometrial cancer: a randomized multi center study. BMC Cancer 9:23
17. Nienhuijs S, de Hingh I (2009) Conventional versus LigaSure hemorrhoidectomy for patients with symptomatic hemorrhoids. Cochrane Database Syst Rev (1):CD006761. doi:10.1002/14651 858.CD006761.pub2

18. Tou S, Malik AI, Wexner SD, Nelson RL (2011) Energy source instruments for laparoscopic colectomy. Cochrane Database Syst Rev (5):CD007886. doi:10.1002/14651858.CD007886.pub2

19. Macario A, Dexter F, Sypal J, Cosgriff N, Heniford BT (2008) Operative time and other outcomes of the electrothermal bipolar vessel sealing system (LigaSure) versus other methods for surgical hemostasis: a meta-analysis. Surg Innov 15(4):284-291 